mentioned that embryonic bodies cultured in the presence of rotenone were smaller than those lacking AIF, suggesting subtle differences between the acute complex I defect induced by rotenone (that might affect homeostatic systems in the cell other than respiration) and the chronic defect induced by the AIF knockout.

These results illustrate the difficulty to separate the two functions of AIF, first as a mitochondrial redox enzyme required for normal respiratory function and, second, as a factor that can participate in the apoptotic execution phase, after its translocation to the nucleus. The observation that AIF participates in cavitation-associated apoptosis ${ }^{9}$ implicitly suggested that cavitation would depend on the lethal action of AIF. Based on recent insights on AIF biology, however, it is possible that at least some of the lethal processes that were interrupted by the removal of AIF from the experimental system actually were suppressed due to defective bioenergetic and/or redox metabolism. As a result, we recommend the use of rotenone as an internal control in experiments, in which the apoptosis-modulatory effects of human or mouse AIF are assessed. Only when rotenone fails to modify the apoptotic response, the interpretation that the lethal (rather than the vital) action of AIF is involved in the process can be maintained.

Knock-in mutations that affect only the lethal function of AIF yet do not interfere with its metabolic activity in mitochondria are being prepared in several laboratories, ${ }^{2}$ and the use of such mutants will yield important insights into the contribution of AIF to lethal signal transduction processes. Such knock-in mutations will also help to decipher the contribution of AIF, alone or in combination with mutations of the apoptosome components, to programmed cell death in physiological and pathological cell death.
Acknowledgements. This work has been supported by a special grant from the Ligue Nationale contre le Cancer (to GK and ND), as well as grants from the European Commission (Trans-Death to GK). OF received a fellowship from the Ligue contre le Cancer.

\section{O Feraud ${ }^{1}$, N Debili $^{1}$, JM Penninger ${ }^{2}$ and G Kroemer $^{\star, 3}$}

1 INSERM U790, Institut Gustave Roussy PR1, Villejuif, France;

2 IMBA, Institute of Molecular Biotechnology of the Austrian Academy of Sciences, Vienna, Austria and

${ }^{3}$ CNRS-FRE2939, Institut Gustave Roussy, Villejuif, France

* Corresponding author: G Kroemer, CNRS-FRE2939 Institut Gustave Roussy, Pavillon de Recherche 139 rue Camille-Desmoulins F-94805 Villejuif, France. Tel: + 331421160 46; Fax: + 331421160 47;

E-mail: kroemer@igr.fr

1. Lorenzo HK et al. Cell Death Differ 1999; 6: 516-524.

2. Modjtahedi N et al. Trends Cell Biol 2006; 16: 264-272.

3. Klein JA, Ackerman SL. J Clin Invest 2003; 111: 785-793.

4. Vahsen $\mathrm{N}$ et al. EMBO J 2004; 23: 4679-4689.

5. Apostolova $\mathrm{N}$ et al. Cell Death Differ 2006; 13: 354-357.

6. Green DR, Kroemer G. Science 2004; 305: 626-629.

7. Parrish JZ, Xue D. Mol Cell 2003; 11: 987-996.

8. Urbano A et al. EMBO J 2005; 24: 2815-2826.

. Joza N et al. Nature 2001; 410: 549-554.

10. Wang $X$ et al. Science 2002; 298: 1587-1592.

11. Wissing $S$ et al. J Cell Biol 2004; 166: 969-974.

12. Liou AK et al. Faseb J 2005; 19: 1350-1352.

13. Park MT et al. Blood 2005; 105: 1724-1733.

14. Artus $\mathrm{C}$ et al. Oncogene 2006, in press.

15. Panka DJ et al. Cancer Res 2006; 66: 1611-1619.

16. Sancho $P$ et al. Apoptosis 2006; 11: 673-686.

17. Yuan CQ, Li YN, Zhang XF. Biochem Biophys Res Commun 2004; 317: 1108-1113.

18. Wang $\mathrm{H}$ et al. J Neurosci 2004; 24: 10963-10973.

19. Yu SW et al. Science 2002; 297: 259-263.

20. Gallego M-A et al. Oncogene 2004; 23: 6282-6291.

21. Coucouvanis E, Martin GR. Cell 1985; 83: 279-287.

22. Joza N et al. Mol Cell Biol 2005; 25: 10261-10272.

23. Brown D et al. Proc Natl Acad Sci USA 2006; 103: 9918-9923.

24. Chance B et al. J Biol Chem 1963; 238: 418-431.

\title{
Broad-spectrum caspase inhibitors: from myth to reality?
}

\author{
Cell Death and Differentiation (2007) 14, 387-391. doi:10.1038/sj.cdd.4402044; published online 29 September 2006
}

Dear Editor,

The Nomenclature Committee on Cell Death has recently warned the scientific community when using terms such as 'caspase-dependent versus caspase-independent cell death' to classify apoptotic versus nonapoptotic cell death. ${ }^{1}$ We strongly agree with this recommendation and we want to add a word of caution concerning the use of broad-spectrum caspase inhibitors in elucidating apoptotic pathways.

The pioneer work in the 1960s by Dr. Robert Smith has established proteolysis by cysteinyl proteinases as a major physiological and pathological process. ${ }^{2}$ To date, 14 mammalian caspases have been identified from the discovery in 1993 that the Caenorhabditis elegans ced-3 gene encoded a homolog of the human interleukin- $1 \beta$-processing enzyme. ${ }^{3} \mathrm{~A}$ striking feature of caspases is their specific ability to recognize particular short peptide sequences and to cleave after an aspartate residue, which is unique among mammalian proteases (except for the serine protease granzyme B). The introduction of an aldehyde group at the C-terminus of such sequences resulted in the generation of reversible inhibitors, whereas chloromethylketone, diazomethylketone, acyloxymethylketone, fluoromethylketone or phenoxymethylketone at this position created inhibitors that irreversibly inactivated caspases through a covalent thioether adduct with the cysteine of the active site. ${ }^{2,4-9}$ First generations of peptidic 'pan-caspase' inhibitors and caspase activity probes were developed in the 1990s by Enzyme Systems Products. These 
inhibitors are often methylated at the extremity of the aspartate lateral chain, thus allowing improved cellular retention when cleaved by endogenous esterases. The development of cell-permeable peptide-based caspase inhibitors provides valuable tools for in vitro and in vivo studies of apoptosis and establishment of the role of caspases in defined stimulus cell death systems. A growing body of evidence supports the contribution of caspase-mediated apoptotic mechanisms in several in vitro and in vivo cell death systems related to a plethora of diseases. ${ }^{2-4,10}$ In the present letter, we want to bring a cautionary note to authors who have not observed any cytoprotective effects when using 'pancaspase' inhibitors, and who only interpret that as resulting from a caspase-independent cell death pathway. This conclusion may sometimes be open to criticism because one may underestimate three major pitfalls: (i) 'pan-caspase' inhibitors are not (systematically) able to inhibit all caspases; (ii) they do not share similar efficiency and specificity profiles against caspases; (iii) they may inhibit other cysteine proteases. To show this, we have focused our demonstration on a side-by-side comparison of various qualities of the widely used fluoromethylketone-based caspase inhibitors (Boc-Dfmk, Z-VAD-fmk) and the more recent 2,6-difluorophenoxymethylketone-based inhibitor, Q-VD-OPH.

The difluorophenoxymethylketone-based broad-spectrum caspase inhibitor Q-VD-OPH is particularly less cytotoxic than fluoromethylketone-based inhibitors against a large panel of human tumor and primary cells but rather equitoxic against prostate and colon carcinoma cells (Supplementary Figure 1a, b). High doses of Boc-D-fmk or Z-VAD-fmk induce necrotic-like features, possibly related to fluoromethylketone conversion into fluoroacetate, which inhibits aconitase ${ }^{2,11}$ (Supplementary Figure 1b, c). In vivo toxic effects of Z-VADfmk have also been reported and may be partly explained by the endogenous production of toxic fluoroacetate, especially in the liver, ${ }^{2,11}$ thus resulting in liver inflammatory cell foci (Supplementary Figure 1d). In contrast, we found that all evaluated organs were normal in Q-VD-OPH-treated mice (Supplementary Figure 1d), suggesting a lack of toxicity of the difluorophenol-leaving group (correlating with reference 19).
Interestingly, the oxamyl dipeptide IDN-6556, which shares structural similarities with Q-VD-OPH, was reported to be safe and well tolerated (up to $10 \mathrm{mg} / \mathrm{kg} /$ infusion for a single dose) in a phase I clinical trial. ${ }^{2,9,12}$ It should be recalled that the development of preclinical fmk-based inhibitors was halted because of hepatotoxicity and dogs dying during acute toxicity studies. ${ }^{2,4}$

We have compared the efficacy of Q-VD-OPH versus ZVAD-fmk or Boc-D-fmk as a cell death inhibitor against various apoptotic stresses. Boc-D-fmk was equally or less potent than Z-VAD-fmk in preventing apoptotic hallmarks (Figure 1a; Supplementary Figure 2a, b). Strikingly, Q-VD$\mathrm{OPH}$ decreased caspase-dependent cell death more significantly than did Z-VAD-fmk and Boc-D-fmk ${ }^{13-15}$ (Figure 1a; Supplementary Figure 2a, b). Cellular effectiveness of Q-VD$\mathrm{OPH}$ is thought to be related to the replacement of the aminoterminal benzyloxycarbonyl (Z) into quinoline moiety (Q) that renders peptides more hydrophobic and enhances cell membrane permeability. This may partially explain why $Q$ VD-OPH induces a much marked inhibition of caspases-3, -8 and -9 in various models (Figure 1a; Supplementary Figure $2 \mathrm{a}-\mathrm{c}$ ), and significantly prevents cell death in high-density cortical neuron cultures withdrawn in serum (Figure 1a). ${ }^{13}$

Pioneer work has clearly demonstrated that 'pan-caspase inhibitors' do not inhibit all caspases equally and should be considered as 'poly-caspase' inhibitors. ${ }^{6,16,17}$ Boc-D-fmk is a poor inhibitor of caspases-2, -5, -6, -10, contrary to Z-VADfmk and Q-VD-OPH (Figures 1b-c). Except for caspase-4, in vitro caspase activities are efficiently prevented by micromolar concentrations of Z-VAD-fmk and Q-VD-OPH, whereas lower concentrations are efficient for Q-VD-OPH (Figure 1b). Surprisingly, Q-VD-OPH also provides the highest caspase-2 and -6 inhibition (Figure 1c): $I_{50}$ Q-VD-OPH $=80 \pm 15 \mathrm{nM}$ (similar to Z-VDVAD-fmk) versus $200 \pm 15 \mathrm{~nm}$ for Z-VAD-fmk for caspase-2; inhibition curves for caspase- 6 are very similar in the presence of Q-VD-OPH, Z-VAD-fmk or the preferential caspase- 6 inhibitor. These data suggest that Q-VD-OPH is a 'true broad-spectrum caspase inhibitor'. One can say that (P3$\mathrm{P} 2-\mathrm{P} 1 / \mathrm{P} 2-\mathrm{P} 1 / \mathrm{P} 1)$ peptide backbones and N-terminus benzyloxycarbonyl versus quinoline groups may create different

Figure 1 Fluoromethylketone- and 2,6-difluorophenoxymethylketone-based broad-spectrum caspase inhibitors show different in vitro/in vivo specificity profiles and inhibition efficiencies. (a) Comparative cellular effects of Boc-D-fmk, Z-VAD-fmk or Q-VD-OPH. (Upper panel) Dose-dependent cell death inhibition by caspase inhibitors in mouse primary cortical neurons were subjected to 24-h serum deprivation (SD) in the presence of Boc-D-fmk, Z-VAD-fmk or Q-VD-OPH $(n=5-8)$. Cells were stained with 7-AAD and Annexin V-FITC to assess viability loss and phosphatidylserine (PS) membrane exposure, before microscopy and flow cytometry analysis. ${ }^{13}$ (Lower panel) Comparative inhibition of caspase activity. 24-h SD neurons were incubated in the absence or presence of $100 \mu \mathrm{M} \mathrm{Boc-D-fmk,} \mathrm{Z-VAD-fmk} \mathrm{or} \mathrm{Q-VD-OPH.} \mathrm{Neurons}$ exhibiting caspase-3, $-8,-9$ activities were quantified under a fluorescence microscope after corresponding FLICA staining ${ }^{13}(n=5)$. (b) In vitro inhibitory efficiency of Boc-D-fmk, Z-VAD-fmk or Q-VD-OPH. Human recombinant caspases 1-10 $(25 \mathrm{U})$ were preincubated $\left(30 \mathrm{~min}, 37^{\circ} \mathrm{C}\right)$ with 0.25 or $1 \mu \mathrm{m}$ indicated caspase inhibitors (or their respective specific inhibitor for internal control, data not shown) and then mixed with their respective AMC-derived substrate $(n=3 ; \lambda e x=405 \mathrm{~nm} ; \lambda \mathrm{em}=510 \mathrm{~nm})$ Significance Z-VAD-fmk versus Q-VD-OPH (unpaired Student's $t$ test): ${ }^{*} P>0.001,{ }^{\#} P>0.01,{ }^{\$} P>0.05$. (c) Caspase-2 (upper panel) and -6 (lower panel) inhibition curves by fmk- or OPH-based caspase inhibitors. In vitro cleavage activity of caspase-2/-6 was assessed in the presence of increasing concentrations of Boc-D-fmk, ZVAD-fmk or Q-VD-OPH as described in (b) ( $n=3)$. Ac-VEID-cho and Z-VDVAD-fmk inhibit caspase-6 and -2, respectively. (d) Caspase inhibition profile by Boc-D-flmk versus Boc-D-OPH. Decreasing concentrations of Boc-D-fmk versus Boc-D-OPH were tested against human recombinant caspases as described in (b) ( $n=3$ ). (e) Dose-response effects of pan-caspase inhibitors against caspase-2-dependent neuronal cell death. Cell death was estimated after 24-h SD as in (a) $(n=3-8)$. Significance (unpaired Student's $t$ test): ${ }^{*} P=0.031,{ }^{\star \star} P=0.010$. A representative western blot shows procaspase-2 $(52 \mathrm{kDa})$ processing into $35 \mathrm{kDa}$ active form after 24-h SD: 1-control, 2-SD, 3-SD + Boc-D-fmk, 4-SD + Q-VD-OPH. (f) In vivo caspase inhibition by Boc-D-fmk, Z-VAD-fmk or Q-VD-OPH in a cerebral ischemia model. Wistar rats were subjected to permanent middle cerebral artery electrocoagulation combined with left common carotid artery transient occlusion. ${ }^{21}$ Caspase inhibitors $(0.1 \mathrm{mg} / \mathrm{kg}$ ) were administered intrajugularly $1 \mathrm{~h}$ after the ischemic onset. Rats were killed $24 \mathrm{~h}$ later and caspase-3,-7-like (Ac-DEVD-AMC) or caspase-9-like (Ac-LEHDAMC) activities were measured in ischemic ispilateral and contralateral brain hemispheres $(n=3)$. Significance (unpaired Student's $t$ test): ${ }^{\#} P=0.036$, ${ }^{\#} P=0.003$, ${ }^{\star} P=0.034$, ${ }^{\star} P=0.006$. (g) Cross-reactivity of fmk- and OPH-based caspase inhibitors against cathepsins $\mathrm{B}, \mathrm{H}, \mathrm{L}$ and $\mathrm{D}$. Human liver cathepsins (250 ng) were preincubated for $1 \mathrm{~h}$ with inhibitors and then mixed for $2 \mathrm{~h}\left(37^{\circ} \mathrm{C}\right)$ with $50 \mu \mathrm{m}$ z-RR-AMC ( $\left.\lambda \mathrm{ex}=380 \mathrm{~nm} ; \lambda \mathrm{em}=460 \mathrm{~nm}\right)$. E6 $64 \mathrm{~d}(2 \mu \mathrm{m})$ was the internal control for inhibition 
interaction networks within the catalytic pocket that may result in different inhibitory patterns. It is known that caspases absolutely require $\mathrm{P} 1$ aspartic acid for catalysis in the $\mathrm{S} 1$ pocket of active site, but in the absence of comparative SAR and co-crystal X-ray structural analysis it is difficult to assume further interactions/binding modes for other residues. How a
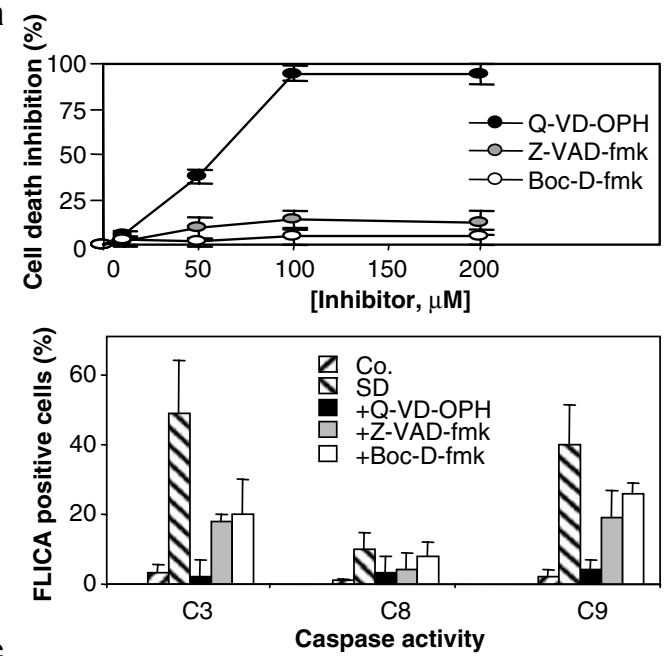

C

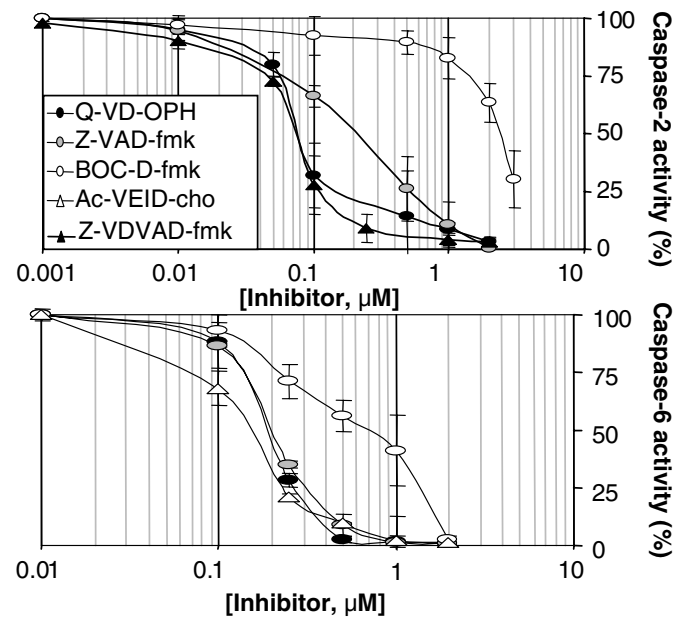

f

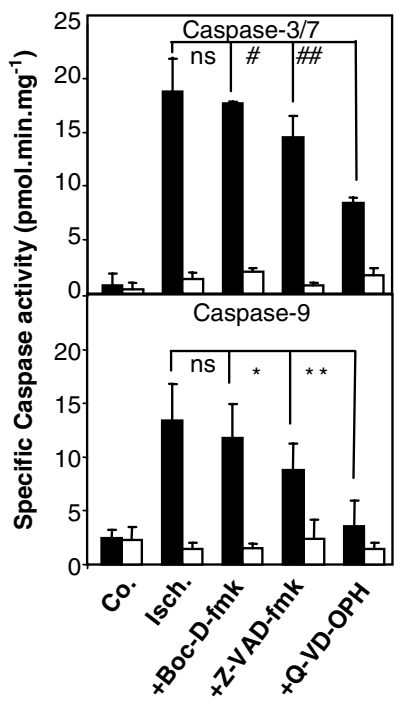

g

Boc-D-fmk 曰 Boc-D-OPH - Q-VD-OPH $\square$ Z-VAD-fmk (1)64d b

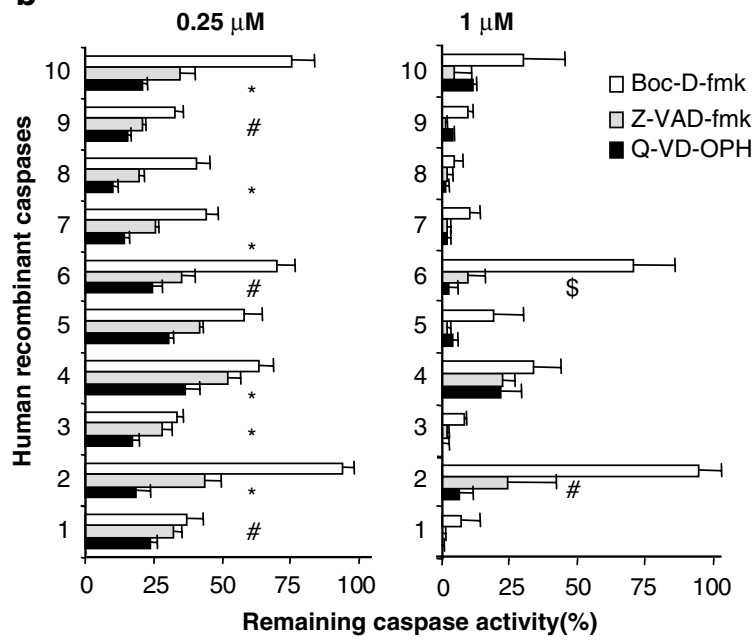

d

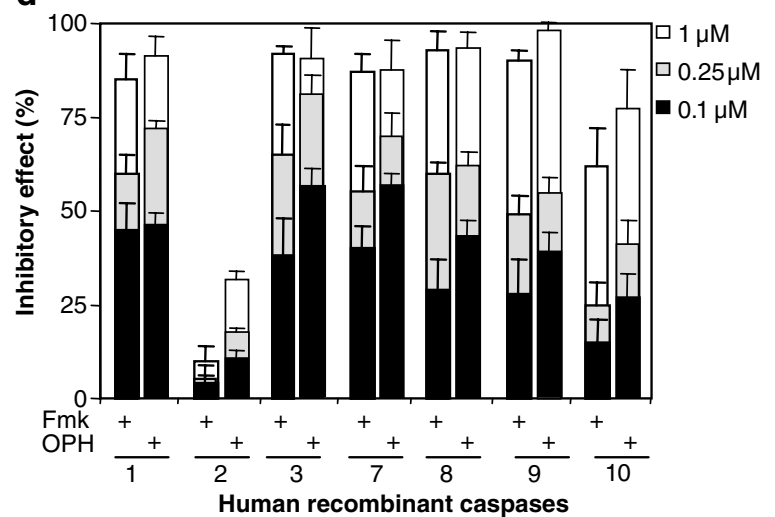

e
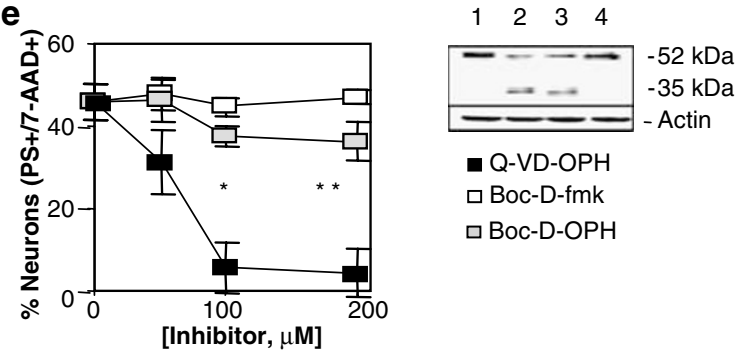

- Q-VD-OPH

$\square$ Boc-D-fmk

$\square$ Boc-D-OPH

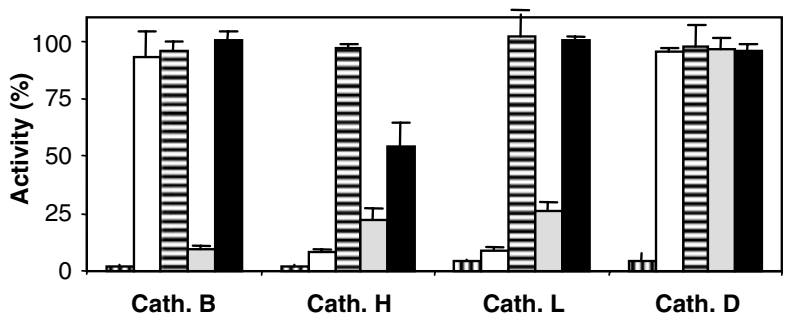


can we explain that Q-VD-OPH (and to a lesser extent Z-VADfmk) and Z-VDVAD-fmk give similar caspase-2 inhibition curves, whereas the X-ray structure of caspase-2 in a complex with Ac-LDESD-CHO has revealed the importance of the $\mathrm{P} 5$ residue to increase ligand recognition and cleavage efficiency $?^{18}$ Which pharmacophore is superior to another independently of the peptide backbone? To try to answer this question, we have synthetized the 2,6-difluorophenoxymethylketone analog of Boc-D-fmk (Supplementary Figure 2d). Comparative Boc-D-OPH versus Boc-D-fmk dose-responses show that lower concentrations of Boc-D$\mathrm{OPH}$ are required to reach caspase inhibition (Figure 1d). This can be explained by the higher hydrophobic nature and nucleofuge properties of $\mathrm{OPH}$. The higher in vitro inhibitory potency of Boc-D-OPH prompted us to check its ability to inhibit in cellula caspase activity. Using siRNA, Z-VDVAD-fmk and Q-VD-OPH, we have recently evidenced the upstream role of caspase-2 in apoptosis of serum-deprived cortical neurons. ${ }^{13}$ In this model, caspase- 2 controls caspase- 9 and -3 activation, ${ }^{13}$ and Boc-D-fmk weakly inhibits caspase-2 activity (not shown). Furthermore, Boc-D-fmk does not prevent procaspase-2 processing, whereas it significantly inhibits downstream caspase-9 and -3 (Figure 1a), without preventing cell death (Figure 1e). Replacement of fmk by OPH results in a modest but significant improvement of in vitro caspase-2 inhibition (Figure 1d), and a related decrease in caspase-2-dependent neuronal death (Figure 1e). This result is not unlike improved caspase inhibition by other fluorophenoxymethylketone-derived oxamyl dipeptides. ${ }^{9}$ It seems that pharmacophore optimization may reinforce caspase recognition and inhibition even if the peptide sequence is not optimal. Surprisingly, Q-VD-OPH but not Boc-D-OPH, is a strong in vitro (Figures 1d) and in cellula (Figure 1e) caspase-2 inhibitor. The quinoline group was initially added to improve cellular penetrability of peptide-based inhibitors. Further modeling studies will show if the highly hydrophobic quinoline may have an unexpected interaction mode with the catalytic pocket of caspases.

Q-VD-OPH also inhibits caspase-1 and caspase-3 in ischemic acute renal failure model. ${ }^{19}$ Chronic administration of Q-VD-OPH induces caspase-3 and caspase-9 inhibition in several mitochondrial toxin models of Parkinson's and Huntington's diseases. ${ }^{20}$ During ischemic brain injury initiated by permanent middle cerebral artery electrocoagulation associated with transient carotid occlusion, caspase-3 activity can be decreased in the lesioned brain hemisphere of rats treated intraperitonally with $10 \mathrm{mg} / \mathrm{kg} \mathrm{Boc-D-fmk.}{ }^{21}$ Here, we show that a single intravenous administration of only $0.1 \mathrm{mg} /$ $\mathrm{kg}$ Q-VD-OPH results in a more pronounced inhibition of caspases-3/-7 and -9 than Boc-D-fmk (not very effective at this dose) or Z-VAD-fmk, demonstrating higher in vivo reactivity and brain penetrability (Figure $1 \mathrm{f}$ ).

Keeping in mind that the fmk pharmacophore was previously shown to interact with non-caspase cysteine proteases, $^{22}$ we experimentally asked whether the $\mathrm{OPH}$ group would be devoid of unspecific interactions. Z-VADfmk inhibited cathepsins $\mathrm{B}, \mathrm{H}^{22}$ and $\mathrm{L}$, whereas Q-VD-OPH does not inhibit cathepsins $B$ and $L$ but slightly inhibits cathepsin $\mathrm{H}$ (Figure 1g). Incubation of Boc-D-fmk and Boc-D$\mathrm{OPH}$ with cathepsins $\mathrm{H}$ and $\mathrm{L}$ clearly demonstrates that fmk, but not $\mathrm{OPH}$, is recognized by these enzymes (Figure 1g). It should be noted that Boc-D-fmk and Z-VAD-fmk exhibit differential inhibitory potency against cathepsin $B$, suggesting that this may be related to the reactivity of the peptide backbone with the catalytic triad of cathepsins (Figure 1g). None of the tested inhibitors affects cysteine-protease calpains (not shown). Granzyme B and the (aspartateprotease) cathepsin $D$ are not inhibited by Boc-D-fmk, BocD-OPH, Z-VAD-fmk or Q-VD-OPH (not shown). These data demonstrate that in contrast to fmk-based inhibitors, OPHbased peptides (at least Boc-D-OPH or Q-VD-OPH) do not cross-react with cathepsins, thus indicating that this new generation of inhibitors only targets the caspase family.

Caspases represent one key family of apoptosis regulators. For more than 10 years, broad-spectrum caspase inhibitors have been used extensively as tools to discriminate caspase-dependent versus independent cell death in physiological and pathological contexts. Comparing several generations of fmk-based and OPH-based caspase inhibitors, we have presented here some data that lead us to propose the following. To determine caspase dependency, experimental design should include both Z-VAD-fmk and QVD-OPH, as the latter has a wider spectrum of action among caspases (being, for instance, a potent caspase-2 and -6 inhibitor, contrary to Z-VAD-fmk). Moreover, each compound can serve as a reciprocal internal control for the other one to exclude pharmacophore- or peptide backbonerelated inhibition of non-caspase proteases (for instance, Z-VAD-FMK inhibits cathepsins $\mathrm{B}, \mathrm{H}$ and $\mathrm{L}$ but $\mathrm{Q}-\mathrm{VD}-\mathrm{OPH}$ does not). It should be added that fluorophenoxymethylketone-based inhibitors seem more efficient than fluoromethylketone-based caspase inhibitors in most in vitro or in cellula models (for instance, Q-VD-OPH provides the highest caspase- 3 inhibition in in vitro caspase assays and several in cellula death models). Finally, safety and biodisponibility considerations may strongly favor the use of $\mathrm{Q}-\mathrm{VD}-\mathrm{OPH}$ in in vivo experimental settings.

Acknowledgements. We are indebted to Pr. S Renolleau for methodology transfer and training in ischemic models. We thank Dr. A BorgneSanchez and $\mathrm{R}$ Greenstein for having read the manuscript with critical eyes. This work was supported by the Agence Nationale pour la Valorisation de la Recherche (Oseo-ANVAR No. R0209333Q and A0404096Q) and the French Ministry of Research (Bio-Ingenierie Program; No. 01H0476).

\section{Chauvier ${ }^{1, *}, S$ Ankri $^{1}$, C Charriaut-Marlangue ${ }^{2}$, $R$ Casimir $^{1}$ and $E$ Jacotot ${ }^{1}$}

\footnotetext{
1 Theraptosis Research Laboratory, Theraptosis S.A., Pasteur BioTop, Institut Pasteur, 25-28 rue du Docteur Roux, Paris, France and

2 Université Pierre et Marie Curie-Paris 6, UMR-CNRS 7102, 9 quai St-Bernard, Paris, France

* Corresponding author: D Chauvier, Theraptosis Research Laboratory, Theraptosis S.A., Pasteur BioTop, Institut Pasteur, 25-28 rue du Docteur Roux, 75015 Paris, France. Tel: + 331406105 47; Fax: + 331536901 98;

E-mail: dchauvier@theraptosis.com
}

1. Kroemer $\mathrm{G}$ et al. Cell Death Differ 2005; 12: 1463-1467.

2. Van Noorden CJF. Acta Histochem 2001; 103: 241-251.

3. Yuan J et al. Cell 1993; 75: 641-652.

4. Fisher U, Schulze-Osthoff K. Pharmacol Rev 2005; 57: 187-215. 

5. Graybill TL et al. Int J Pept Protein Res 1994; 44: 173-182.
6. Thornberry NA et al. Biochemistry 1994; 33: 3934-3940.
7. Dolle RE et al. J Med Chem 1994; 37: 563-564.
8. Wang $Y$ et al. Bioorg Med Chem Lett 2005; 15: 1379-1383.
9. Linton SD et al. J Med Chem 2005; 48: 6779-6782.
10. Degterev A et al. Oncogene 2003; 22: 8543-8567.
11. Gribble GW. J Chem Educ 1973; 50: 460-462.
12. Valentino KL et al. Int J Clin Pharmacol Ther 2003; 41: 441-449.
13. Chauvier D et al. Apoptosis 2005; 10: 1243-1259.
14. Caserta TM et al. Apoptosis 2003; 8: 345-352.
15. Chen J et al. Apoptosis 2005; 10: 1131-1142.
16. Garcia-Calvo M et al. J Biol Chem 1998; 273: 32608-32613.
17. Fuentes P, Salvesen GS. Biochem J 2004; 384: 201-232.
18. Schweizer A. J Biol Chem 2003; 278: 42441-42447.
19. Melnikov Y et al. J Clin Invest 2002; 110: 1083-1091.
20. Yang L et al. Neurobiol Dis 2004; 17: 250-259.
21. Joly L-M et al. J Cereb Blood Flow Metab 2004; 24: 124-131.
22. Schotte $\mathrm{P}$ et al. FEBS Lett 1999; 442: 117-121.

Supplementary Information accompanies the paper on Cell Death and Differentiation website (http://www.nature.com/cdd) 